\title{
Potential common radiation problems for components and diagnostics in future magnetic and inertial confinement fusion devices
}

\author{
J. Alvarez ${ }^{\mathrm{a}, *}$, R. Gonzalez-Arrabal ${ }^{\mathrm{a}}$, A. Rivera ${ }^{\mathrm{a}}$, E. Del Rio ${ }^{\mathrm{a}}$, D. Garoz ${ }^{\mathrm{a}}$, E.R. Hodgson ${ }^{\text {b }}$, F. Tabares ${ }^{\mathrm{b}}$, \\ R. Vila ${ }^{\text {b }}$ M. Perlado ${ }^{a}$ \\ a Instituto de Fusión Nuclear, UPM, José Gutiérrez Abascal, 2, Madrid, Spain \\ ${ }^{\mathrm{b}}$ EURATOM/CIEMAT Fusion Association, Madrid, Spain
}

A B S T R A C T

Keywords:

Nuclear fusion

Inertial confinement

Magnetic confinement

Radiation damage

Materials

Diagnostics
This work aims at identifying common potential problems that future fusion devices will encounter for both magnetic and inertial confinement approaches in order to promote joint efforts and to avoid duplication of research. Firstly, a comparison of radiation environments found in both fusion reaction chambers will be presented. Then, wall materials, optical components, cables and electronics will be discussed, pointing to possible future areas of common research. Finally, a brief discussion of experimental techniques available to simulate the radiation effect on materials is included.

\section{Introduction}

Engineers and scientists must meet the challenge of producing a long-lasting and clean source of energy such as fusion. In order to achieve fusion energy, there are currently two basic approaches: magnetic (MC) and inertial confinement (IC). Although these methodologies are radically different in the way they achieve the plasma densities and required temperatures to produce fusion, they face common radiation damage issues. The harsh environment that both fusion reaction chambers have to withstand consists of high fluxes of neutrons, gamma, X-rays and energetic light ions (see references in Section 2). Even when differences in the radiation pulse length ( $\sim 200 \mathrm{~ns}$ for IC and hundreds of seconds for MC) affect the material response, similar thermo-mechanical and atomistic effects are found. Accumulation of tritium and activation are also a common matter of concern. These undesirable effects have to be understood and controlled so that the proper functioning of the facility is assured.

Large experimental facilities are required to test chamber components under such extreme radiation conditions. Joint development and use of certain facilities can be beneficial for both, MC and IC communities. In the same way, R\&D in new materials, diag- nostic components and remote handling designed for one approach can be beneficial for its counterpart.

The aim of this paper is to identify common potential problems in $\mathrm{MC}$ and IC fusion to promote collaboration, reduce costs, and avoid research duplication.

\section{Radiation fluxes}

Basically, the production of fusion and radiation in IC is cyclic in nature (pulsed) whereas MC fusion takes place in near steady state conditions (long pulses). However, the most remarkable radiation events in MC appear as prompt emissions: (i) type I edge localized modes (ELM) and (ii) disruptions [1,2]. Table 1 compares the rough values of these MC events at the ITER divertor to those of a typical $154 \mathrm{MJ}$ direct drive target in IC [3]. In a direct drive IC fusion explosion, apart from the penetrating neutrons, the major part of the energy goes to three species: fusion product $\alpha$-particles and (non-burnt) debris D and T ions (see Fig. 1).

From table I one can see that deposited energies in the MC events are much larger than those in IC. However, peak powers are higher in IC. In order to see the effect of such a deposition of energy on the chamber components one should consider the kinetic velocity of the impinging particles. Since in IC the incoming ions are much more penetrating than in MC, energy is deposited along a larger depth, reducing appreciably the energy and power density on the material (see Fig. 2). The pulse duration and heat diffusion must also be taken into account. This is frequently expressed 
Table 1

Optimistic conditions assumed for ITER divertor and for a typical direct drive target (yield 154 MJ) [2,3].

\begin{tabular}{|c|c|c|c|c|c|c|c|}
\hline & & Time (s) & $\begin{array}{l}\text { Deposited energy } \\
\left(\mathrm{MJ} \mathrm{m}^{-2}\right)\end{array}$ & $\begin{array}{l}\text { Power } \\
\left(\mathrm{MW} \mathrm{m}^{-2}\right)\end{array}$ & $\begin{array}{l}\text { Heat flux parameter } \\
\left(\mathrm{MW} \mathrm{m}^{-2} \mathrm{~s}^{-1 / 2}\right)\end{array}$ & $\begin{array}{l}\text { Particle } \\
\text { energy (ev) }\end{array}$ & $\begin{array}{l}\text { Particle flux } \\
\left(\mathrm{m}^{-2} \mathrm{~s}^{-1}\right)\end{array}$ \\
\hline \multirow[t]{3}{*}{ Divertor } & Steady state & 1000 & - & 15 & - & $1-30$ & $<10^{24}$ \\
\hline & ELM & $0.2 \times 10^{-3}$ & 1 & $5 \times 10^{3}$ & 70 & $1-30$ & $<10^{24}$ \\
\hline & Disruptions & $1 \times 10^{-3}$ & 20 & $2 \times 10^{4}$ & 600 & $1-30$ & $<10^{24}$ \\
\hline \multirow[t]{2}{*}{ Direct target } & $\alpha$-Particles & $200 \times 10^{-9}$ & 0.03 & $1.5 \times 10^{5}$ & 70 & $2.1 \times 10^{6} \mathrm{avg}$ & $1 \times 10^{25}$ \\
\hline & DT debris & $1.5 \times 10^{-6}$ & 0.06 & $4 \times 10^{4}$ & 50 & $150 \times 10^{3}$ avg. & $2 \times 10^{22}$ \\
\hline
\end{tabular}

$\mathrm{E}(\mathrm{MJ})$

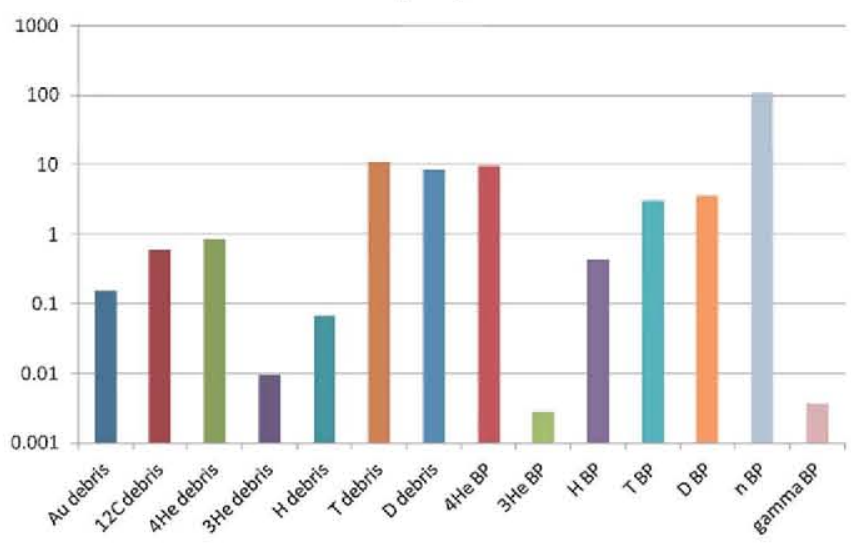

Fig. 1. Energy distribution for different particles produced by a direct drive target of $154 \mathrm{MJ}$. BP stands for burn products.

by means of the so-called heat flux parameter which is defined as $H=E(\Delta t)^{-1 / 2}$, where $E$ represents the deposited energy and $\Delta t$ the deposition time [4]. $H$ values for different MC and IC processes are given in Table 1. Similar H values are shown to induced similar thermal effects, so materials designed to withstand heat loads from ELMs are expected to thermally hold up IC explosions and vice versa. So far we have compared both MC and IC radiation fluxes in terms of thermal loads into the materials that in turn may produce deleterious thermo-mechanical effects such as roughening, cracking or melting. Nevertheless, ion-matter interactions resulting in defect production, i.e. ion implantation, play also an important role. Understanding the ion-induced phenomena is not straightforward due to synergistic effects stemming from the simultaneous implantation of several ion species. Note that although the ingredients for defect-driven phenomena exist in both $\mathrm{MC}$ and IC, the implantation species, energies and fluxes drastically differ.

To date, the effect of gamma rays and neutrons (see Table 2) also represent a common problem mostly on damage of optical components (see below) and activation issues. In the future, when fusion reactors work as electrical power stations, the effect of neutron displacements (100 dpa/year) will also be determinant for the survivability of the reactors.

\section{Common material issues for divertor/first wall}

In addition to carbon-based materials, currently, the most promising material for the MC divertor and IC armor is tungsten [6-8].

From a thermo-mechanical point of view neither of the sofar studied materials can withstand the most disadvantageous MC conditions, e.g. disruptions. For W, analytical solutions of one-dimensional heat equation under disruption conditions yield temperatures exceeding $30,000 \mathrm{~K}$ on the surface (ignoring melting and vaporization) which would lead to unavoidable mass loss and damage. In the case of ELMs in MC and He fusion products in IC, temperatures would raise above $3000 \mathrm{~K}$, close to the melting point and above the thresholds for cracking formation. The IC community is working on developing alternative materials that enable the use of dry wall chambers with reduced radius $(R<5-6 \mathrm{~m})$. The new materials must fulfill certain requirements: (i) large surface area to accommodate the thermal load over a larger volume; (ii) high thermal conductivity to impede excessive heating due to reduced thermal removal [9].

From an atomistic point of view, IC W-based armor materials present a serious problem regarding He nucleation in vacancy clusters that, in turn, leads to blistering and exfoliation of the material with fatal mass losses. This problem is also an issue in MC. In both cases modeling of blistering is not trivial due to the synergistic effects taking place. A way of minimizing this problem can be achieved by developing: (iii) porous materials to facilitate the release of He and other light species; (iv) self-healing materials i.e. nanocrystals in which vacancies easily migrate to grain boundaries reducing the formation of large vacancy clusters and thus He nucleation.
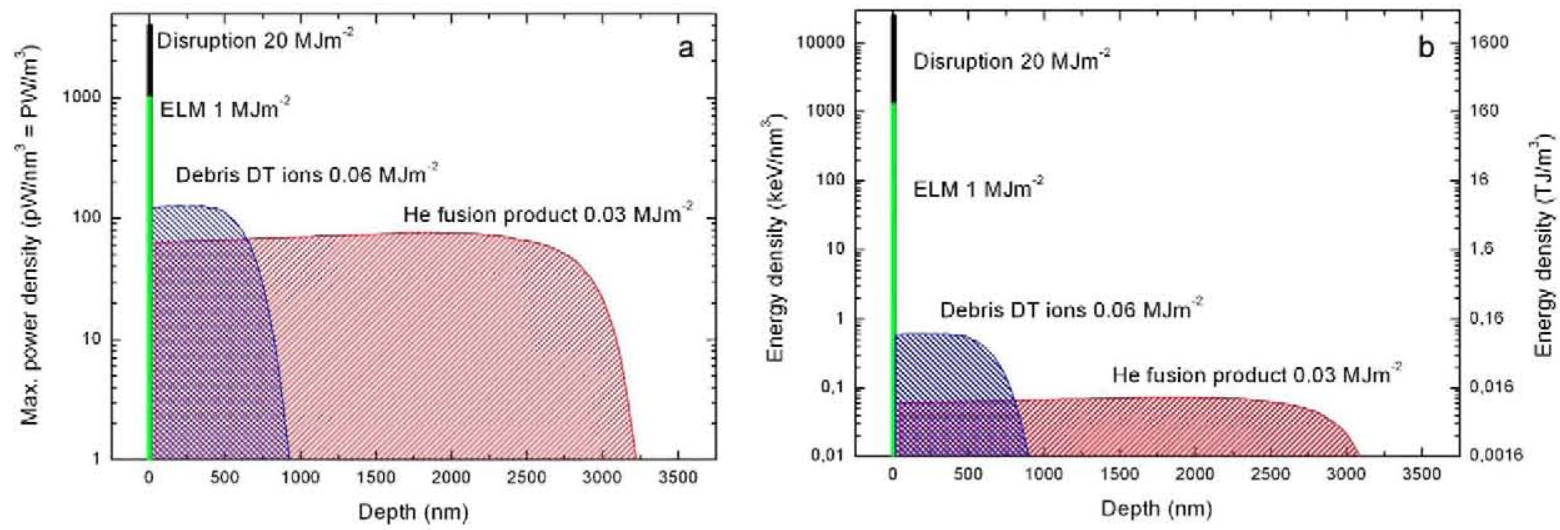

Fig. 2. (a) Power density as a function of depth in a W sample for different MC and IC conditions. (b) Energy density deposited in a W sample as a function of depth. 
Table 2

Rough neutron fluxes and gamma ray doses assumed for ITER [5] and a inertial direct drive target (yield 154 M]),

\begin{tabular}{|c|c|c|c|}
\hline First wall & Neutron flux $\left(\mathrm{m}^{-2} \mathrm{~s}^{-1}\right)$ & Neutron fluence (end of life) $\left(\mathrm{m}^{-2}\right)$ & Dose rate gammas (Gy/s) \\
\hline ITER & $3 \times 10^{18}$ & $3 \times 10^{25}$ & $2 \times 10^{3}$ \\
\hline IC & $3 \times 10^{16} \mathrm{~m}^{-2}$ shot equivalent to $10^{25}$ & $10^{20}$ & $10^{11}$ \\
\hline
\end{tabular}

Tritium retention [10] has been found to be an important issue in $\mathrm{MC}$ when C-based materials are used, since its chemical erosion and subsequent hydrocarbon formation and co-deposition can lead to levels of tritium retention beyond those permitted by legal regulations. Further studies are needed to establish whether tritium retention is an issue in IC dry chambers with C-based armor.

\section{Common components and materials for diagnostic systems}

Four possible areas have been indicated where collaboration on diagnostic systems preparations could be of mutual benefit, namely optical components (fibres, windows, and mirrors), MI (mineral insulated) cables, electronics, and neutron diagnostics [5]. Of these, the most relevant common issue is the numerous optical transmission components, in particular windows and fibres, required for diagnostics in the case of MC, and for both operation and diagnostics for IC. For such materials/components, the obvious main concern comes from the gamma and neutron radiation, and additionally in the case of windows, surface degradation and contamination. Although equivalent to surface degradation, the problem of laser damage, debris and shrapnel will not be considered here as this is limited to IC.

At the present time suitable radiation resistant window materials, in general, fused silica such as KU1, with acceptable radiation induced absorption (RIA) and luminescence (RIL) are available for MC applications [11]. However this is only true when the windows are placed in well shielded regions with reduced dose rates $\left(<100 \mathrm{~Gy} / \mathrm{s},<10^{-9} \mathrm{dpa} / \mathrm{s}\right)$, which requires the use of high reflectivity dielectric mirrors. In the case of IC the orders of magnitude higher radiation flux during each pulse may well make RIL a problem despite heavy shielding. It is therefore of mutual advantage to investigate means to effectively reduce the RIL, both by suitable choice of material, and by examining the possibility of window operation at elevated temperatures $(\approx 600 \mathrm{~K})$ to quench the emission [12]. Furthermore high temperature operation has the added advantage of reducing the RIA due to both ionization and displacement damage. RIA for high displacement dose $\left(\geq 10^{-4} \mathrm{dpa}\right)$ remains a limitation for both IC and MC systems. A joint effort by both communities to investigate the possibility of high temperature operation and in situ annealing as a means of increasing the useful lifetime for window components should be considered. Window surface degradation is another area of common concern; such degradation not only reduces the transmission, but also lowers the laser damage threshold and can lead to failure [13]. Of course in situ protection shutters will help to reduce the problem and could be jointly pursued; however collaborative work on in situ detection methods coupled with surface cleaning by means of low energy (sub-threshold) laser pulses would be more beneficial for long term operation [14].

The simplification of diverse diagnostic systems offered by optical fibres, is off-set by their high radiation sensitivity [11]. Three aspects limit the use of fibres in a high radiation environment. Firstly the length itself means that acceptable levels of RIA and RIL for limited thickness windows become intolerable in the long fibres. Success in producing fibres with low initial absorption and reduced RIA, has in general been met with a corresponding increase in the RIL. And finally the fabrication process itself plays an important role, as it can introduce detrimental defects into an otherwise radiation resistant boule material. Within the MC community extensive $R \& D$ on candidate fibre materials has been carried out, with both fluorine-doped and pure-silica being investigated, as well as hydrogen loading which can substantially reduce RIA [15]. As with windows, RIA and RIL in fibres strongly depend on irradiation temperature, and in general are less at higher temperatures due to reduced defect stability and quenching. With all this in mind collaboration on necessary radiation testing, jacketing suitable for high temperature operation, exchange of candidate fibres, as well as a joint programme to define and control fibre fabrication parameters would be of considerable interest to both fusion communities, and most beneficial.

Finally a brief mention should be made of possible collaborations on cables and electronics. MC systems will use mainly robust and radiation tolerant MI cables, ideal for low voltage and low frequency applications, as well as being vacuum compatible, and suitable for high temperature operation [11]. However due to the limited bandwidth of MI cables, for many IC applications standard PTFE/CH high frequency dielectric cables will be employed [11]. Hence in this field the only area of mutual interest would be an interchange of data concerning the influence of RIC (radiation induced conductivity), RIEMF (radiation induced electromotive force), and possibly TIEMF (temperature induced electromotive force) on measured signals, as well as potential mitigation methods. In the case of electronics present activities in both communities rely heavily on COTS (commercial off-the-shelf) components, redundant design, distance and heavy shielding. Early MC activity related to remote handling requirements included radiation testing of electronic components, and it soon became clear that advances in available electronic components towards miniaturization rendered them far more susceptible to radiation damage. One possible solution included basic circuit design employing more radiation resistant discrete components (transistors), work along such lines would be of benefit to both communities.

\section{Experimental facilities to simulate irradiation conditions}

Both fusion communities are aware of the necessity to test materials and components. However at present no entirely suitable irradiation testing facility exists, hence experiments are performed in fission reactors and particle accelerators, as well as gamma and $\mathrm{X}$-ray sources, in an attempt to reproduce the real operating conditions [11]. Especially difficult is to simulate high ion flux scenarios as those presented in Section 2. One may use infra-red facilities or focused electron beam to mimic high thermal fluxes. However, serious limitations exist and no considerations on atomistic effects arise from that [4]. Several plasma gun facilities have been developed by the MC community to study plasma-wall interactions under realistic conditions [16]. On the other hand, much less effort has been undertaken by the IC community to mimic the short and high flux ion and X-ray pulses present in IC reactors. The radiationinduced damage which can be generated under these conditions might significantly differ from that produced under continuous irradiation conditions. In this respect, it is of particular interest the ion facility RHEPP [6] and the X-ray sources Z pinch and XAPPER $[17,18]$ in the USA.

One must simulate the neutron and gamma radiation field i.e. displacement and ionization damage rates, radiation environment i.e. vacuum and temperature and also operating conditions such 
as applied voltage, or mechanical stress. For insulators in situ testing is essential to determine whether or not the required physical properties are maintained during irradiation. For example electrical conductivity which can increase many orders of magnitude due to ionizing radiation, or optical windows which may emit intense radio-luminescence. The fluxes, energy spectrum, dpa to ionization and $\mathrm{He}$ ratios achieved with neutrons and gammas from experimental fission reactors are not those of a fusion reactor. In addition, the irradiation channels are small and inaccessible, limiting the possibility for in situ testing and experiments in vacuum.

Particle accelerators, on the other hand, are ideal for carrying out in situ experiments in high vacuum and at well controlled temperatures due to easy access and localized radiation field. High levels of displacement damage and ionization can be achieved with little or no nuclear activation. It is however in the non-nuclear aspect of the radiation field where their disadvantage is evident. A further serious disadvantage is the limited irradiation volume and particle penetration depth. This means that only small thin samples or components can be tested. Moreover, in order to simulate the synergetic effect taking place in fusion radiation environments, double and triple beams facilities are needed. Since the number of these facilities all around the world is very low, major efforts have to be done in joining research to share out existing data and to propose common experiments.

However despite the limitations, full advantage is being taken not only of fission reactors and particle accelerators, but also ${ }^{60} \mathrm{Co}$ gamma irradiation facilities and even X-ray sources. The use of such widely different radiation sources can be justified as long as the influence of the type of radiation on the physical parameter of interest is known.

\section{Conclusions}

Joint research is not only desirable but also beneficial for both MC and IC communities in their goal to achieve energy by fusion. The present work has identified potential common issues in which such collaboration naturally appears. Although the way of producing fusion and the time scale of the fusion events are different for both fusion approaches, the similar radiation environment of the chambers housing the reactions poses common threats. One of the weak points in the fusion race is related to the development of materials able to satisfactorily withstand the thermo-mechanical and atomistic effects happening in the MC divertor and IC first wall. Currently, new materials with similar advanced properties (large surface, high thermal conductivity, porous, ...) are being developed for those purposes. Common work on this topic will be a step forward in our way to fusion.

The damage due to neutrons and gamma rays, mostly on optical components and diagnostic devices also paves the way for a joint investigation. Test facilities for radiation damage are scarce and expensive and sometimes restrict their validity to particular cases. This justifies the development of common experimental proposals and the design of future facilities suitable for both communities, as in the case of a neutron source.

\section{References}

[1] A.R. Raffray, G. Federici, A. Hassanein, D. Haynes, Fusion Eng. Des. 63-64 (2002) 597.

[2] A. Herrmann, T. Eich, S. Jachmich, M. Laux, P. Andrew, A. Bergmann, et al., J. Nucl. Mater. 313-316 (2003) 759.

[3] http://aries.ucsd.edu/ARIES/WDOCS/ARIES-IFE/SPECTRA/.

[4] J. Linke, F. Escourbiac, I.V. Mazul, R. Nygren, M. Roedig, J. Schlosser, et al., J. Nucl. Mater. $367-370$ (2007) 1422.

[5] J.L. Bourgade, A.E. Costley, R. Reichle, E.R. Hodgson, W. Hsing, V. Glebov, et al., Rev. Sci. Instrum. 79 (10) (2008) F304.

[6] T. Hirai, H. Maier, M. Rubel, P. Mertens, R. Neu, E. Gauthier, et al., Fusion Eng. Des. 82 (2007) 1839

[7] V. Barabash, A. Peacock, S. Fabritsiev, G. Kalinin, S. Zinkle, A. Rowcliffe, et al., J. Nucl. Mater. 367-370 (2007) 21.

[8] J.D. Sethian, A.R. Raffray, J. Latkowski, J.P. Blanchard, L. Snead, T.J. Renk, et al., J. Nucl. Mater. 347 (2005) 161.

[9] T.J. Renk, P.P. Provencio, T.J. Tanaka, C.L. Olson, R.R. Peterson, J.E. Stolp, et al., J. Nucl. Mater. 34 (2005) 266.

[10] J. Roth, E. Tsitrone, T. Loarer, V. Philipps, S. Brezinsek, A. Loarte, et al., Plasma Phys. Controlled Fusion 50 (2008) 103001

[11] G. Vayakis, E.R. Hodgson, V. Voitsenya, C.I. Walker, Fusion Sci. Technol. 53 (2008) 699 .

[12] A. Moroño, E.R. Hodgson, J. Nucl. Mater. 258 (1998) 1889.

[13] P. Martin, A. Moroño, E.R. Hodgson, J. Nucl. Mater. 307 (2002) 1260.

[14] A.V.Gorbunov, N.V. Klassen, D. Orlinski, K.Y.Vukolov, Fusion Eng. Des. 74 (2005) 815 .

[15] B. Brichard, A.F. Fernandez, H. Ooms, F. Berghmans, M. Decreton, A. Tomashuk, et al., J. Nucl. Mater. 329 (2004) 1456

[16] EFDA (09) 41/4.2 Issue 3, 29 September 2009.

[17] T.J. Tanaka, G.A. Rochau, R.R. Peterson, C.L. Olson, J. Nucl. Mater. 347 (2005) 244

[18] J.F. Latkowski, R.P. Abbott, R.C. Schmitt, B.K. Bell, J. Nucl. Mater. 347 (2005) 255. 